\title{
Clinical highlights: messages from Munich
}

\author{
loannis Vogiatzis ${ }^{1,2}$, Maurizio Marvisi ${ }^{3}$, Johan Coolen ${ }^{4}$, Stefano Gasparini ${ }^{5,6}$, \\ Katerina M. Antoniou ${ }^{7}$, Bjorn Stallberg ${ }^{8}$, Anders Bjerg ${ }^{9}$, Felix J.F. Herth ${ }^{10,11}$ and \\ Enrico Clini ${ }^{12}$
}

Affiliations: ${ }^{1}$ First Dept of Respiratory Medicine, Pulmonary Rehabilitation Unit, Sotiria Hospital, National and Kapodistrian University of Athens, Athens, Greece. ${ }^{2}$ First Dept of Critical Care Medicine, Pulmonary Rehabilitation Centre, National and Kapodistrian University of Athens, Athens, Greece. ${ }^{3}$ Dept of Internal Medicine and Pneumunology, Clinica Figlie di S. Camillo, Cremona, Italy. ${ }^{4}$ Dept of Radiology, University Hospital Gasthuisberg, Leuven, Belgium. ${ }^{5}$ Dept of Biomedical Sciences and Public Health, Polytechnic University of the Marche Region, Ancona, Italy. ${ }^{6}$ Pulmonary Diseases Unit, Azienda Ospedaliero-Universitaria Ospedali Riuniti, Ancona, Italy. ${ }^{7}$ Dept of Thoracic Medicine Department and Laboratory of Molecular and Cellular Pneumonology, Medical School, University of Crete, Heraklion, Crete, Greece. ${ }^{8}$ Dept of Public Health and Caring Sciences, Family Medicine and Preventive Medicine, Uppsala University, Uppsala, Sweden. ${ }^{9}$ University of Gothenburg, Krefting Research Centre, Gothenburg, Sweden. ${ }^{10}$ Dept of Pulmonary and Critical Care Medicine, University of Heidelberg. Heidelberg, Germany. ${ }^{11}$ Translational Lung Research Centre Heidelberg, Heidelberg, Germany. ${ }^{12}$ Dept of Medical and Surgical Sciences, University of Modena-Reggio Emilia, Modena, Italy.

Correspondence: loannis Vogiatzis, National and Kapodistrian University of Athens, Department of Critical Care Medicine, 3 Ploutarhou Str, 10675, Kolonaki, Athens, Greece. E-mail: gianvogaphed.uoa.gr

ABSTRACT This article reviews a selection of presentations in the area of clinical problems that were presented at the 2014 European Respiratory Society International Congress in Munich, Germany. We review the most recent and relevant topics of interest in the area of clinical respiratory medicine, encompassing novel reports and studies that are of particular interest to healthcare professionals. Topics ranging from basic science to translation research are presented and discussed in the context of the most up-to-date literature. In particular, the reviewed topics deal with chronic obstructive pulmonary disease and asthma, idiopathic pulmonary fibrosis (pathogenesis and therapy), advances in functional chest imaging, interventional pulmonology, pulmonary rehabilitation, and chronic care.

@ERSpublications

Clinical highlights from the 2014 ERS International Congress in Munich, Germany http://ow.ly/MwUMg

Received: March 202015 | Accepted: April 292015

Conflicts of interest: None declared.

Copyright $\odot$ ERS 2015. This article is open access and distributed under the terms of the Creative Commons Attribution Non-Commercial Licence 4.0. 


\section{Introduction}

The 2014 European Respiratory Society (ERS) International Congress was held in Munich, Germany (fig. 1). This meeting is the largest in the world in the area of respiratory medicine, exceeding 22000 attendees. In 2014, a total of 4390 abstracts were presented (80\% acceptance rate), of which, around 20\% dealt with specific clinical problems and were referred to the ERS Clinical Assembly.

\section{Methods}

Abstracts were selected from presentations at the ERS 2014 International Congress that were available on line. The selection was based on the individual group chairs' preferences and evaluation of the perceived importance of each relevant topic.

\section{Clinical problems}

Several new studies were presented at the Congress regarding pathogenesis, phenotyping and clinical/ therapeutic applications in chronic respiratory patients. Here, we report intriguing insights in the area of chronic obstructive pulmonary disease (COPD) and other chronic conditions.

\section{Chronic obstructive pulmonary disease}

Polverino et al. [1] studied the role of B-cell activating factor (BAFF) in COPD. They quantified BAFF expression in B- and T-cells in bronchoalveolar lavage (BAL) and blood samples of 38 COPD patients, 17 smokers and 20 nonsmokers by using flow cytometry. They found that BAFF expression was increased in B- but not in T-cells in BAL and, to a lesser extent, in blood samples from COPD patients. Furthermore, BAFF levels in BAL B-cells were inversely correlated with the severity of airflow limitation, suggesting that B-cell expansion, mediated by BAFF, may be a critical event in the pathogenesis of the disease. The problem of endothelial dysfunction was tackled by VUKIC DUGAC et al. [2] in two phenotypically distinct groups of frequent and infrequent COPD exacerbators. Von Willebrand factor, as a marker of endothelial dysfunction, and C-reactive protein and fibrinogen, as markers of systemic inflammation, were measured in 117 consecutive subjects; a significantly higher increase of all markers in frequent than infrequent exacerbators was found. The presence of allergic sensitisation (serum specific IgE for inhaled antigens) is associated with worse baseline characteristics and clinical course in humans with COPD. SUZUKI et al. [3] designed a 5-year prospective observational cohort study including 268 COPD patients. 67 (25\%) subjects had allergic sensitisation; this phenotype was not associated either with worse baseline characteristics (post-bronchodilator forced expiratory volume in $1 \mathrm{~s}$ (FEV1), computed tomography (CT) emphysema score and quality of life) or with clinical course (annual decline in FEV1 and exacerbation frequency).

The inflammatory pattern in the stable state and during exacerbation of COPD patients was reported by SINGH et al. [4], who specifically investigated the levels of tumour necrosis factor (TNF)- $\alpha$ released level by monocyte-derived macrophages (MDMs) stimulated by bacterial load. MDMs were cultured in inert beads or with a heat-killed Haemophilus influenzae or Streptococcus pneumoniae and the TNF- $\alpha$ release level was measured by ELISA. They found that H. influenzae is more pro-inflammatory than S. pneumoniae in both clinical conditions. However, a significant increase in TNF- $\alpha$ release level from the stable to exacerbation state was seen in response to $S$. pneumoniae only. These data guarantee future research about this response and the relationship with phagocytosis.

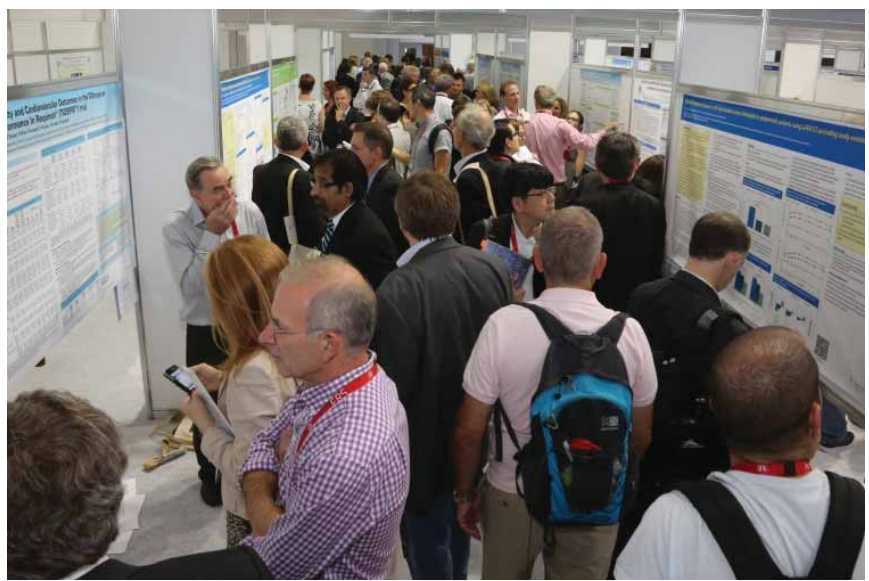

FIGURE 1 A thematic poster session at the 2014 European Respiratory Society International Congress in Munich, Germany. Image courtesy of Michael Buxbaum, The International Center for Documentary Arts, Chicago, IL, USA. 
BнAтT et al. [5] faced the topic of the paradoxical response to bronchodilators in COPD, as defined by at least $12 \%$ or $200 \mathrm{~mL}$ reduction in $\mathrm{FEV} 1$ and/or forced vital capacity (FVC) post-bronchodilator. They included 9986 subjects from a large multicentre study (COPD-GENE); the paradoxical response was seen in $4.54 \%$ of patients, was similar in those with COPD and in smokers without airflow obstruction, and was independently associated with worse dyspnoea, higher BODE index and a greater frequency of exacerbations. These subjects were younger, current smokers and African-Americans. The paradoxical response to bronchodilators may therefore represent a novel COPD phenotype.

The problem of dealing with self-management in the population of exacerbated COPD patients was tackled by Zwerink et al. [6]. Over 2 years of follow-up, the self-treatment of exacerbations did not lead to a decrease in the number or severity of exacerbations; however, it was shown to lead to fewer pulmonary physician consultations, with no increase in hospitalisations or emergency department visits. An interesting study from UK. [7] demonstrated that lower limb muscle mass measured by ultrasound predicts the risk of rehospitalisation following admission for acute exacerbations of chronic respiratory disease.

\section{Other lung diseases}

Fibrosing mediastinitis is a rare but potentially fatal disease. Westerly et al. [8] evaluated the role of rituximab in a small cohort of patients receiving a 2-month tapered dose of prednisone. Circulating B-cells were depleted in all patients and all had favourable therapeutic responses (fig. 2).

LichtBlau et al. [10] preliminarily studied the feasibility and long-term effects of switching pulmonary arterial hypertension (PAH) patients showing intolerable side-effects due to sildenafil to tadalafil. In six out of 13 subjects, switching to tadalafil was feasible and resulted in better tolerated side-effects and a more stable clinical course, achieving improvements in symptoms, 6-min walking distance, stable echocardiographic findings, lung function and N-terminal pro-brain natriuretic peptide levels during a follow-up of $11 \pm 3$ months. In five out of 13 patients, adverse events occurred under tadalafil as well, so therapy with phosphodiesterase (PDE)-5 inhibitors was discontinued. In two patients, sildenafil treatment was successfully restarted after an intermittent switch to tadalafil. The observations of this study indicate that a transition of PDE-5 inhibitors due to intolerable side-effects is reasonable in approximately half of patients.

Lichtblau et al. [11] investigated the role of stress Doppler echocardiography (SDE) for early detection of $\mathrm{PAH}$ in systemic sclerosis (SSc). They assessed 76 consecutive patients by echocardiography at rest, SDE, and right heart catheterisation at rest and during exercise. The sensitivity of the diagnosis of PAH increased up to $90 \%$ by using SDE, which may be a useful screening tool in patients with SSc. The clinical efficacy of inhaled $\mathrm{N}$-acetylcysteine in a heterogeneous group of patients with interstitial pneumonia (idiopathic pulmonary fibrosis (IPF), nonspecific interstitial pneumonia and connective tissue diseases
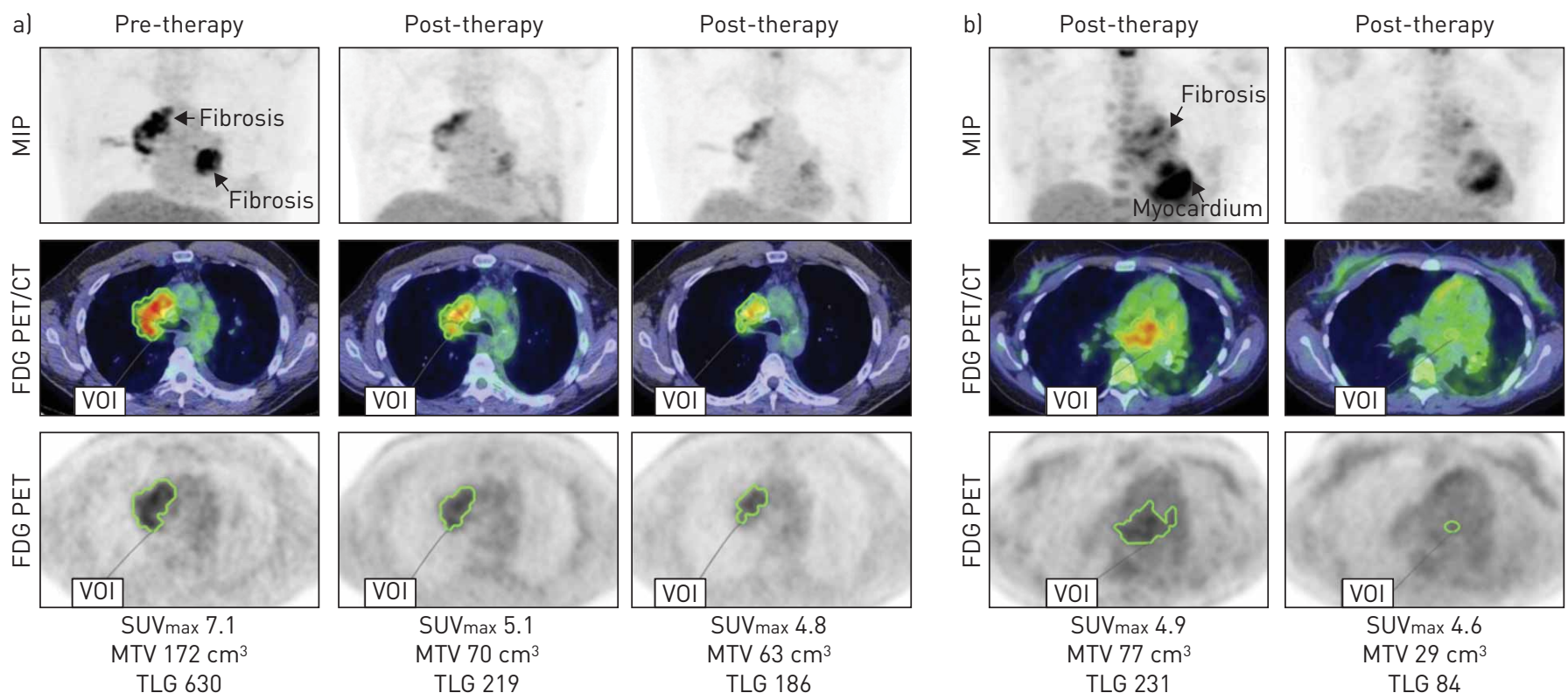

FIGURE 2 Metabolic response by ${ }^{18}$ F-fluorodeoxyglucose (FDG) positron emission tomography (PET)/computed tomography (CT). a) FDG PET/CT for patient 2 at baseline (pre-therapy) and at 6 and 12 months of B-cell depletion (post-therapy). b) FDG PET/CT for patient 3 at baseline (pre-therapy) and 7 months after B-cell depletion (post-therapy). MIP: maximum intensity projection; VOI: volume of interest; SUVmax: maximum standardised uptake value; MTV: metabolic tumour volume; TLG: total lesion glycolysis. Reproduced from [9] with permission from the publisher. 
(CTD)) was studied by OKUDA et al. [12]. Interestingly enough, they were able to show a lower rate of reduction of FVC in subject treated over 6 months $(-19 \mathrm{~mL})$ when compared with a similar follow-back period $(-127 \mathrm{~mL})$.

\section{Rehabilitation and chronic care}

Important advances in the area of rehabilitation and chronic care emerged demonstrating significant benefits on essential outcomes such as functional capacity, symptoms, health-related quality of life and healthcare utilisation. Accordingly, evidence was provided supporting the effectiveness and feasibility of rehabilitation in patients with COPD, obstructive sleep apnoea, asthma, metastatic lung cancer or malignant pleural mesothelioma, and interstitial lung disease (ILD), and in patients undergoing single- or double-lung transplantation. Family- and community-based rehabilitation programmes were shown to be effective in terms of improving functional capacity, assessed by the 6-min walk test, and quality of life. An increasing number of studies performed in a conventional outpatient setting corroborated the positive impact on daily physical activity assessed by triaxial accelerometers. The minimal clinically important difference (MCID) in essential outcome measures of pulmonary rehabilitation, such as daily physical activity, chronic dyspnoea and the incremental shuttle walk (ISW) test was also reported.

Along these lines, in a retrospective analysis, SCHNEeberger et al. [13] evaluated the effects of pulmonary rehabilitation in 550 patients with COPD or ILD immediately after single- or double-lung transplantation. While health-related quality of life improved to a similar extend in patients after both single- and double-lung transplantation, exercise capacity improved significantly more in the latter population. In the study by OLIVIER et al. [14], 71 patients treated for lung cancer or malignant pleural mesothelioma with chemotherapy benefited from a rehabilitation programme combining electrostimulation or exercise training with therapeutic education and psychosocial care, by exhibiting significant improvements in quality of life and exercise capacity. In adults with moderate or severe asthma, aerobic training was shown to decrease bronchial hyperresponsiveness and serum proinflammatory cytokines, while improving clinical control [15].

Interesting results were presented also on nonconventional pulmonary rehabilitation strategies. As pulmonary rehabilitation often neglects the potential contribution of the family, MARQUES et al. [16] investigated the role of family members participating in psychoeducation together with COPD patients. This family-based programme was shown to enhance the coping resources of the family in managing COPD (fig. 3), and to be feasible in that it did not interfere with conventional rehabilitation and its widely recognised patient benefits. A community-based rehabilitation regimen incorporated in a self-management programme [17] showed a sustained increase in daily physical activity despite a fall in maximal exercise capacity over 2 years.

Improvements in daily physical activity (monitored by total energy expenditure and active energy expenditure by a triaxial accelerometer) were documented after a 10-week outpatient pulmonary rehabilitation programme in patients with moderate to severe COPD regardless of the magnitude of improvement in functional capacity assessed by the 6-min walk test [18]. The effectiveness of pulmonary

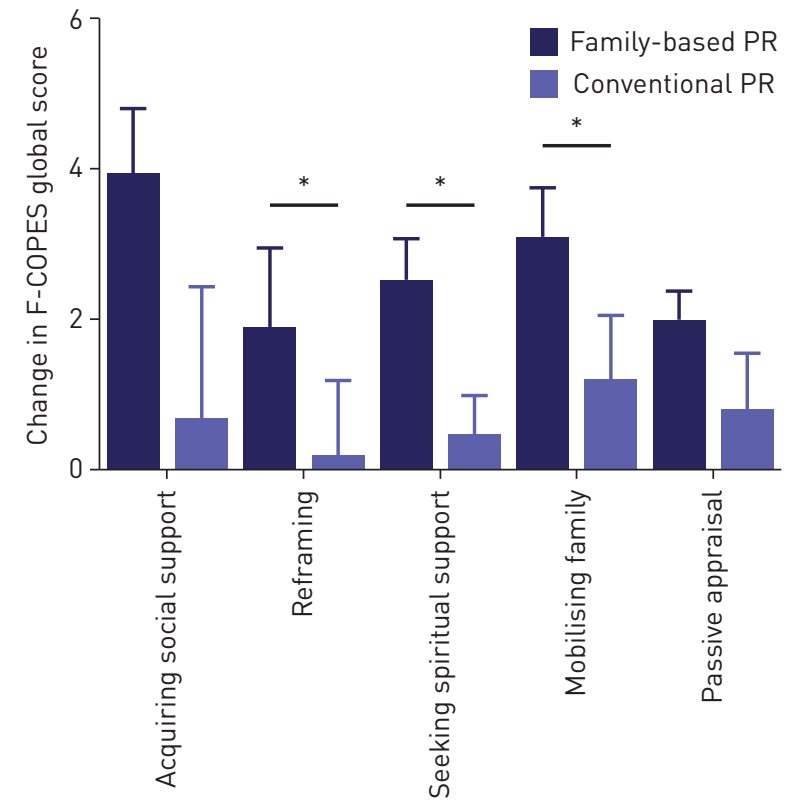

FIGURE 3 A family-based pulmonary rehabilitation (PR) programme enhanced the coping resources of the families of chronic obstructive pulmonary disease patients. F-COPES: Family Crisis Oriented Personal Scales. Reproduced from [16] with permission from the authors. 
rehabilitation in terms of improvement in daily movement intensity assessed by accelerometry was reported following employment of a high-intensity interval exercise-training regimen [19].

While the study by DEMEYER et al. [20] corroborated the efficacy of pulmonary rehabilitation in improving daily step counts in COPD, importantly, it also determined the magnitude of minimal important difference (MID) of this outcome, which ranges between 576 and 1181 steps. The MID of the ISW test in patients with COPD with a low baseline ISW distance was determined by Nolan et al. [21] to be $40 \mathrm{~m}$. JoHnson et al. [22] addressed the question of whether the MCID in chronic dyspnoea intensity should be based on absolute or relative measures and concluded that it should be expressed as a relative reduction of $15-20 \%$ from baseline measurements.

\section{Chest imaging}

Even in the era of fused imaging studies, two presentations at the Congress showed that traditional single-modality observer studies are still fundamental. In a retrospective analysis of 100 spirometer-controlled CT scans at total lung capacity (TLC), Vos et al. [23] demonstrated that bronchopulmonary anatomical variations are significantly frequent, i.e. nine anatomical variations were observed in $68 \%$ of patients. In another observational study, HOYER et al. [24] showed that standard and basic chest radiograph is still relevant in chest pain patients suspected of acute coronary syndrome; indeed, when combined with clinical and laboratory information, it can rule out most differential diagnoses.

The role of lung imaging continues to move from purely morphological to a more functional approach, which is nicely illustrated in sarcoidosis and COPD. Besides diagnostic achievements, technological advances were also presented at the imaging sessions concerning these diseases. First, high-resolution CT (HRCT) seems to be useful in the diagnosis of sarcoidosis, as many radiological patterns can be visualised [25]. Interstitial thickening and nodular opacities are the most common parenchymal alterations while paratracheal and symmetric hilar lymph node enlargement are the most common mediastinal abnormalities on HRCT [26]. PAPIRIs et al. [27] examined, in 35 patients, the role of ${ }^{18} \mathrm{~F}$-fluorodeoxyglucose positron emission tomography/CT for the detection of thoracic and extrathoracic manifestations of sarcoidosis. They detected few but clinically meaningful cases of cardiac, musculoskeletal and ocular sarcoidosis, leading to more comprehensive therapeutic management. Cardiac sarcoidosis, occurring in up to $30 \%$ of patients with pulmonary sarcoidosis, was investigated by STANTON et al. [28]. They identified that a mid-wall pattern of late gadolinium enhancement is associated with a hyperintense myocardial signal on imaging, indicating active inflammation, and with the presence of significant arrhythmias.

Reduced left ventricular cardiac performance is not only observed in the presence of severe emphysema but also in hyperinflation due to abnormal lung function in COPD. Using lung function tests and Doppler echography, Poraknonko et al. [29] found that COPD patients with hyperinflation (inspiratory capacity/ TLC $\leq 0.25$ ) have impaired left ventricular diastolic filling. GALBAN et al. [30] used parametric response mapping (PRM) of inspiratory/expiratory CT scans to monitor the longitudinal chances in COPD subtypes. They found good correlations between the mathematical model simulations and PRM analysis of CT in 76 patients, suggesting that functional small airway disease (i.e. COPD with nonemphysematous tissue) may serve as a transitional phase from normal parenchyma to emphysema. This PRM model could thus allow characterisation of COPD patients based on disease subtyping.

Pulmonary function testing provides only limited information on regional lung characteristics and, due to issues of irradiation, the CT modality is not the first choice of repeated COPD monitoring examination. Knowing that relaxation times in lungs depend on tissue composition including the blood volume fraction, JoBst et al. [31] validated tissue and oxygen-enhanced (OE) mapping against first-pass perfusion magnetic resonance imaging (MRI) in 20 COPD patients. Both of these correlated well with lung perfusion in the different Global Initiative for Chronic Obstructive Lung Disease (GOLD) stages. In particular, OE mapping contributed to the differentiation of hypoxic vasoconstriction from genuine perfusion impairment.

Imaging with hyperpolarised helium-3 or xenon-129 (enriched 86\%) gas ventilation MRI has been proposed for direct visualisation of the local heterogeneity due to match/mismatch and delayed ventilation, but these noble gas techniques are too expensive to achieve sufficient image quality in the routine practice. PARRA-Robles et al. [32] presented an alternative and relatively inexpensive method using natural-abundance polarised xenon-129 (enriched 26\%) gas with a regulatory-approved polariser.

A few more innovative abstracts on functional imaging were presented and focussed at the level of alveolar microstructures, where MRI and IPF came increasingly under the spotlight. Figure 4 illustrates a three-dimensional picture model derived from helium-3 gas diffusion MRI projected on micro-CT images to generate models of acinar airways, which allowed computer simulations illustrating the complexity of the lung microstructure [32].

HART et al. [33] applied the MRI phenomenon that the relaxation time in fibrotic lung is significantly shorter than in healthy lung. Using the magnitude of this time shortening, the effect was significantly 
a)

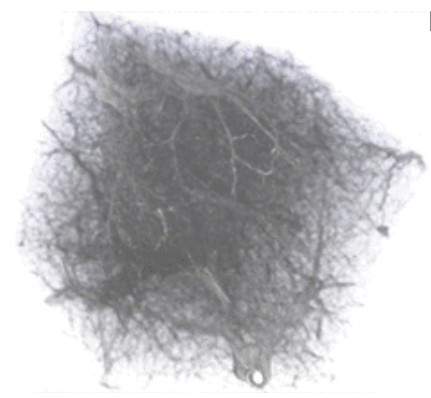

c)

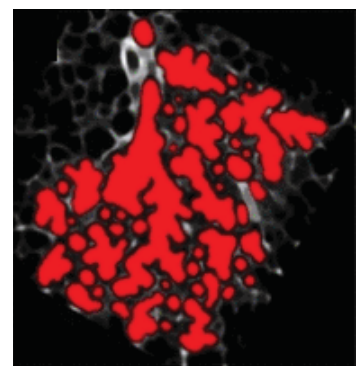

b)

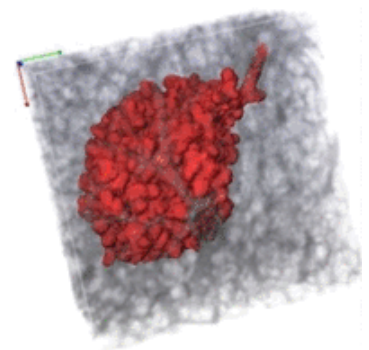

d)

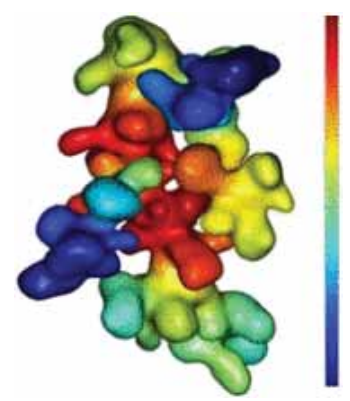

FIGURE 4 a) Micro-computed tomography data corresponding to a helium-3 magnetic resonance imaging voxel size of $7 \times 7 \times 7 \mathrm{~mm}$ and b) the segmented single acinus volume. c) A cross-section of segmented airspace mask and d) a three-dimensional helium-3 diffusivity distribution map obtained from the computer simulation. Reproduced from [32] with permission from the authors.

smaller in IPF patients than controls, indicating less efficient gas exchange in the pulmonary blood pool of these patients.

In regards to the feasibility of noninvasive measurement of lung septal thickness with hyperpolarised xenon-129 MRI in healthy volunteers and subjects with IPF, STEWART et al. [34] found that xenon-129 MRI is sensitive detecting small changes in lung septa with the ageing of the lung that cannot be identified by usual pulmonary function tests. Taken together, these new data encourage the use and further research of MRI not only for functional assessment of COPD-associated diseases but also for other pulmonary parenchymal diseases such as IPF, where MRI could also objectify the therapeutic advances towards IPF management.

Finally, the group of DE BACKer et al. [35] presented a combination of pulmonary function tests and functional respiratory imaging. This research group found that disease progression in IPF is correlated with reduced lobar volume and airway impedance and enlarged airway volume. Further studies need to confirm these initial and innovative observations.

It is likely that the cooperation between different specialists and researchers (pathologists, pneumologists, radiologists, physicist, engineers, statistician, etc.) will make imaging-based scientific progress possible in the near future.

\section{Interventional pulmonology}

\section{Endoscopic treatments for obstructive lung diseases}

Promising data on novel procedures, as well as further insights on already established techniques, were the major topics in the field of endoscopic treatments for obstructive lung diseases.

SLEBos et al. [36, 37] presented the 1-year outcomes of a first-in-human feasibility study on targeted lung denervation (TLD) in patients with moderate to severe COPD. Similar to anticholinergic drugs, TLD is intended to reduce acetylcholine by the endoscopic ablation of parasympathetic nerves along the main bronchi, using a catheter-based lung denervation system. TLD was performed in 22 moderate to severe COPD patients, showing $>15 \%$ FEV1 reversibility to ipratropium, at two different energy doses (20 or $15 \mathrm{~W}$ ) in a staged fashion (two procedures with rigid bronchoscopy 30 days apart). Overall, sustained improvements in bronchodilation, exercise endurance and quality of life were observed at 1-year follow-up off bronchodilators, especially in the 20-Watt group [36]. However, data on safety were not completely reassuring, as 16 dose-independent serious adverse events were reported. In particular, two severe adverse events were reported, namely one sudden death and one gastric paresis [36, 37]. Encouraging data on feasibility and long-term outcomes were reported also by VALIPOUR et al. [38], who performed TLD in both lungs during a single procedure at $15 \mathrm{~W}$ in $15 \mathrm{COPD}$ patients with the same eligibility criteria. TLD showed a similar efficacy of long-acting muscarinic antagonists, as FEV1 and 6-min walking test off tiotropium at 1-year follow-up were comparable to the baseline values on tiotropium. Notwithstanding, 
the benefit of such procedures in terms of cost-effectiveness profile, as well as the role of long-term structural changes of the airway wall, need to be further investigated in large studies.

Due to the increasing diffusion of lung volume reduction treatments for advanced emphysema in daily practice, a large amount of noteworthy experiences, mainly referring to coils and valve systems, was presented at the Congress. New insights in lung volume reduction coil (LVRC) treatment included the evidence of a significant improvement of chest wall asynchrony at optoelectronic plethysmography in a well-designed, although small, controlled study [39] and the suggestion of favourable effects on ventilation/ perfusion relationship, assessed by iodine maps acquired by dual-energy CT [40]. The available data at 3-year follow-up showed an acceptable safety profile and an overall decline in benefits over time, although there was still a significant number of responders. However, the high proportion of missing visits (42\% of patients did not attend the 3-year follow-up) could have introduced selection bias to outcome estimates [41].

The efficacy of endobronchial valve treatment was further confirmed in a high-quality experimental setting [42], as well as in less "selected" subgroups of patients usually excluded from clinical trials, i.e. subjects with $\mathrm{FEV}_{1}<20 \%$ of predicted or with established pulmonary hypertension (PH) [43, 44]. Even in these patients, data on short-term safety still showed a relatively high occurrence of spontaneous pneumothorax, although this seemed to be reduced by strict bed rest for the 2 days after the procedure [45].

Among the novel approaches for advanced emphysema treatment, encouraging results come from the experience of biological lung volume reduction using a catheter-based autologous blood injection. These preliminary data suggest an interesting cost-effectiveness profile [46]. The outcome at 1 year in 85 patients with homogenous emphysema treated by bronchoscopic nitinol LVRCs was reported by MARCHETTI et al. [47]. They showed that LVRCs, compared with patients treated by medical therapy alone, improved lung mechanics (hyperinflation and air trapping) and exercise tolerance, thus suggesting a role for this procedure in the most advanced homogeneous emphysema.

\section{Diagnostic procedures}

GUPTA et al. [48] addressed the issue of accuracy and safety of endobronchial ultrasound (EBUS)transbronchial needle aspiration (TBNA) over conventional TBNA in patients suspected of having sarcoidosis; even though, individually, EBUS-TBNA has the highest diagnostic yield, it should be combined with transbronchial lung biopsy to achieve optimal results. Notwithstanding, complementary approaches [49], including rapid cytological analysis [50], have been raised in recent reports.

Interesting and new data on complementary techniques for transbronchial aspiration procedures were presented at ERS Congress in 2014. RozMAN et al. [51] investigated the role of ultrasound elastography (EG) combined with EBUS-TBNA in the diagnostic work-up of mediastinal lymph nodes. EG allows assessment of biomechanical properties of tissue by a strain ratio measurement, allowing differentiation between benign and malignant lesions. At strain ratios $\geq 8$, the accuracy of malignancy prediction in 66 mediastinal lymph nodes from 27 suspected lung cancer patients was $89.4 \%$, showing it to be a reliable tool for the selection of biopsy targets.

Herth et al. [52] presented a first-in-human trial on the safety and yield of bronchoscopic transparenchymal nodule access, a promising new image-guided technique for diagnostic flow in patients with small solitary lung nodules. A straight-line path from an airway wall entry point to the target lesion was calculated using software, and an access sheath and a 15-gauge stylet were advanced through parenchymal tissue under fused CT/fluoroscopic guidance. Overall, the procedure was feasible, safe and accurate (yield 83\%), and encourages clinicians to plan further experiences in larger populations.

\section{Interstitial lung diseases}

At the 2014 ERS Congress, updates on the diagnosis, prognosis and/or treatment of both sarcoidosis and IPF, the two most relevant ILDs, were presented. Additional interesting information came from studies of systemic diseases associated with lung fibrosis.

\section{Sarcoidosis}

In patients with sarcoidosis, an easy staging system has recently been suggested to stratify patients and identify those at high clinical risk by integrating the composite physiological index with HRCT measurements [53].

When considering the natural course and prognosis of this lung disease, gene polymorphisms seem to make an important contribution to the clinical phenotype. The presence of the TNFA 308A variant allele, which relates to TNF- $\alpha$ production, has been associated with a better prognosis [54]. In addition, patients without the TNFA 308A variant allele (GG genotype) had a three-fold higher responses to TNF inhibitors (adalimumab or infliximab) [54]. 
The therapy with adalimumab (40 mg weekly for 45 weeks), a fully human anti-TNF monoclonal antibody, has been compared with infliximab in terms of both efficacy and safety in patients with refractory pulmonary sarcoidosis [55]. Out of the 11 patients completing the study, seven stabilised their lung volumes (FVC) and four improved. Moreover, adalimumab was associated with less use or withdrawal of systemic steroids and immunomodulators in $27 \%$ of patients. Ustekinumab and golimumab are monoclonal antibodies that specifically inhibit interleukin (IL)-12 and IL-23, and TNF- $\alpha$, respectively. Although well tolerated, neither treatment with ustekinumab nor golimumab showed efficacy in pulmonary sarcoidosis [56].

\section{Idiopathic pulmonary fibrosis}

IPF is a chronic, progressive and rare ILD of unknown aetiology with a low survival rate. A global IPF registry would facilitate a better understanding of disease molecular and cellular biology and behaviour, as well as identification of regional variations in diagnostic and management patterns that will help establish better treatment approaches [57]. The ASCEND trial [58] was designed to clarify the contrasting results of the earlier studies (CAPACITY) with oral pirfenidone in IPF. In this trial, for the first time, pirfenidone significantly improved progression-free survival and slowed the decline of FVC compared with placebo at 52 weeks.

The effect of pirfenidone on lung function and survival was then reported in those patients who were previously randomised to the placebo group in the CAPACITY studies and then received this drug for the first time [59]. Treatment was safe and generally well tolerated, with side-effects consistent with previous reports; most importantly, the decline in FVC was significantly reduced in patients treated with pirfenidone compared with placebo.

The INPULSIS trials are replicate phase III randomised studies comparing the efficacy and safety of the tyrosine kinase inhibitor nintedanib (150 mg twice per day) with placebo in patients with IPF [60]. Nintedanib reduces annual decline in lung function (FVC) by approximately $50 \%$.

\section{Other lung fibrosis}

Safety and efficacy of treatment with bosentan, a dual endothelin-1 receptor antagonist, was reported in patients suffering from confirmed $\mathrm{PH}$ associated with lung fibrosis (idiopathic interstitial pneumonia (IIP)) [61]. This study showed no difference in invasive pulmonary haemodynamics, functional capacity or symptoms between the bosentan and placebo groups over 16 weeks, thus suggesting the uselessness of bosentan in these patients.

Identifying occult CTDs in patients with presumed idiopathic ILDs can be challenging and requires a comprehensive, often multidisciplinary, evaluation. There is much uncertainty and controversy surrounding the suggestive forms of CTD-associated ILD (CTD-ILD) and prospective studies are still needed to provide a better understanding of the natural history of these cohorts. In the setting of CTDs, some measures of ILD disease activity and severity may be confounded by nonpulmonary comorbidities. A core set comprising specific measures in the domains of lung physiology, lung imaging, survival, dyspnoea, cough and health-related quality of life was proposed to be appropriate for use in clinical trials for either CTD-ILD or IPF [62].

A multicentre prospective study of 104 patients diagnosed as having IIPs or newly diagnosed CTD-ILD treated with corticosteroids was conducted [63]. The pattern of usual interstitial pneumonia (by HRCT) and a lower performance status have important prognostic implications in patients with IIPs.

\section{General practice and primary care}

At the 2014 Congress, some interesting data were presented dealing with the diagnosis and management of respiratory diseases at the primary care level.

KосKS et al. [64] identified different phenotypes in a broad spectrum of obstructive airway disease in a primary care population. Data on 13 variables from a structured primary care asthma/COPD service were used to identify clusters using hierarchical clustering. Six distinct groups were thus identified in this primary care population. This might have relevant consequences in terms of both drug and long-care management.

The prevalence of COPD and related risk factors in a noncontinental rural area in Africa was investigated [65]. The authors performed a population-based cross-sectional survey of 588 randomly selected subjects above age of 30 years. Trained local healthcare workers used validated questionnaires and performed spirometry. The prevalence of COPD was $16 \%$, with the majority of both women and men exposed to indoor biomass smoke. In the COPD group, $44 \%$ of the men were current and $24 \%$ former smokers, and among women, $8 \%$ were current and $18 \%$ former smokers. They concluded that the prevalence of COPD in Uganda is relevantly high in lower age groups, exposure to biomass smoke and smoking in men being the two most significant risk factors. 
Jones et al. [66] had assessed the effect of the alternative methods of calculating the GOLD COPD categories on their distribution and future risk of exacerbations (best assessed using the exacerbation frequency) including the new cut-off values. They used data from more than 7000 primary care patients with valid data in the UK. Depending on the method used, the proportion in category D varies from less than 5\% to $46 \%$. The prospective relationship between the number of exacerbations and eosinophilia was studied by PrICE et al. [67] in 13780 general practice patients with a diagnosis of COPD. The authors concluded that blood eosinophilia occurs in stable COPD patients and is associated with a higher exacerbation risk, especially in nonsmokers receiving maintenance treatment. The role of smoking in future exacerbation risk is still to be elucidated.

Regarding the change of individual's characteristics over time, SunDH et al. [68] examined factors associated with change in DOSE index [69] in a 7-year follow-up study performed in COPD patients from primary and secondary care, with data collected in between 2005 and 2012. The conclusion from this study was that heart disease signals patients at risk of worsening their severity when assessed by DOSE.

KRUIS et al. [70] evaluated, in a 24-month cluster randomised controlled trial, the effects of integrated disease management (IDM) in COPD patients compared to usual care. IDM consisted of integrated care including spirometry, assessment of disease burden, exacerbation management, motivational interviewing, physiotherapeutic reactivation and smoking cessation. They found improvement in the level of care integration but failed to record any improvement in the primary outcome (difference in Clinical COPD Questionnaire score at 12 months) compared to usual care.

Finally, in a study from Spain [71], authors evaluated a new model of integrated care in which hospital specialists attend patients in different primary care centres. They assessed the impact of this model on the management of respiratory diseases in a retrospective, observational study comparing two care models in the same cohort of patients: conventional (prior model of care by pulmonologists not integrated into primary care teams) and integrated. They found that the implementation of a respiratory care model integrated into primary care improved the follow up of these patients by reducing the overall number of visits for exacerbations, possibly due to the optimisation of the diagnosis and treatment.

\section{References}

1 Polverino F, Pons J, Iglesias A, et al. BAFF: a self-perpetuating mechanism of B cell activation in COPD. Eur Respir J 2014; 44: Suppl. 58, 2950.

2 Vukic Dugac A, Jakopovic M, Popovic Grl S, et al. Endothelial dysfunction and systemic inflammation in different COPD phenotypes. Eur Respir J 2014; 44: Suppl. 58, 2951.

3 Suzuki M, Konno S, Makita M, et al. Effect of allergic sensitization on clinical course of patients with COPD in the Hokkaido COPD cohort study. Eur Respir J 2014; 44: Suppl. 58, 2956.

4 Singh R, Belchamber K, Mackay A, et al. TNF $\alpha$ release by monocyte-derived macrophages at stable and exacerbation states in COPD. Eur Respir J 2014; 44: Suppl. 58, 2957.

5 Bhatt S, Wells J, Kim V, et al. Paradoxical response to bronchodilators: radiologic correlates and clinical implications. Eur Respir J 2014; 44: Suppl. 58, 4634.

6 Zwerink M, Kerstjens AMH, van der Palen J, et al. Effectiveness of self-treatment of exacerbations in COPD patients: two-year follow-up of the COPE-II study. Eur Respir J 2014; 44: Suppl. 58, 415.

7 Greening N, Harvey-Dunstan T, Williams J, et al. Lower limb muscle mass using ultrasound predicts re-hospitalisation following admission for acute exacerbations of chronic respiratory disease. Eur Respir J 2014; 44: Suppl. 58, 417.

8 Westerly B, Johnson G, Maldonado F, et al. B-lymphocyte targeted therapy for patients with progressive fibrosing mediastinitis. Eur Respir J 2014; 44: Suppl. 58, 4636.

9 Westerly BD, Johnson GB, Maldonaldo F, et al. Targeting B lymphocytes in progressive fibrosing mediastinitis. Am J Respir Crit Care Med 2014; 190: 1069-1071.

10 Lichtblau M, Harzheim D, Ehken N, et al. Safety and long-term efficacy of transition from sildenafil to tadalafil due to side effects in patients with pulmonary arterial hypertension. Eur Respir J 2014; 44: Suppl. 58, 4637.

11 Lichtblau M, Ehiken N, Egelauf B, et al. Stress-Doppler-Echocardiography for early detection of systemic sclerosis associated pulmonary arterial hypertension. Eur Respir J 2014; 44: Suppl. 58, 4641.

12 Okuda R, Ohba T, Nishizawa T, et al. Efficacy of inhaled N-acetylcystein in patients with interstitial pneumonia. Eur Respir J 2014; 44: Suppl. 58, 4500.

13 Schneeberger T, Gloeckl R, Sczepanski B, et al. Differences in pulmonary rehabilitation outcomes in patients after single or double lung transplantation. Eur Respir J 2014; 44: Suppl. 58, P618.

14 Olivier C, Delourme J, Cortot A, et al. A feasibility study of pulmonary rehabilitation at home in patients with lung cancer or malignant pleural mesothelioma treated by (radio-)chemotherapy. Eur Respir J 2014; 44: Suppl. 58, 1714.

15 França-Pinto A, Mendes ARF, Stelmach R, et al. Aerobic training decrease bronchial hyper responsiveness and systemic inflammation in patients with moderate or severe asthma: a randomized controlled trial. Eur Respir $J$ 2014; 44: Suppl. 58, 1707.

16 Marques A, Jácome C, Cruz J, et al. Family-based pulmonary rehabilitation in COPD: a randomized controlled trial. Eur Respir J 2014; 44: Suppl. 58, 418.

17 Zwerink $\mathrm{M}$, van der Palen J, Kerstjens $\mathrm{AMH}$, et al. A community-based exercise programme in COPD self-management: two-year follow-up of the COPE-II study. Eur Respir J 2014; 44: Suppl. 58, 415.

18 Malagrinò L, Vagaggini B, Costa F, et al. Physical activity improved significantly in patients with chronic obstructive pulmonary disease non responders to an outpatient pulmonary rehabilitation program (PRP). Eur Respir J 2014; 44: Suppl. 58, P604. 

levels in COPD. Eur Respir J 2014; 44: Suppl. 58, 1709.

20 Demeyer H, Hornikx M, Camillo AMC, et al. An estimation of the minimal important difference in physical activity for patients with COPD. Eur Respir J 2014; 44: Suppl. 58, 1914.

21 Nolan CM, Canavan JC, Kon SC, et al. The minimum important difference (MID) of the incremental shuttle walk (ISW) in COPD: does baseline ISW influence estimates? Eur Respir J 2014; 44: Suppl. 58, 414.

22 Johnson M, Bland M, Oxberry S, et al. Measuring improvement in dyspnoea: Absolute or relative?. Eur Respir J 2014; 44: Suppl. 58, P4744.

23 Vos W, Volkovych S, Van Holsbeke C, et al. Nomenclature of broncho-pulmonary anatomy: 60 years later. Eur Respir J 2014; 44: Suppl. 58, 548.

24 Hoyer N, Steffensen I, Schoos M, et al. Routine chest radiograph is still valuable in patients admitted for acute non-traumatic chest pain, an observational study. Eur Respir J 2014; 44: Suppl. 58, 4966.

25 Criado E, Sanchez M, Ramirez J, et al. Pulmonary sarcoidosis. Typical and atypical manifestations at high-resolution CT with pathologic correlation. Radiographics 2010; 30: 1567-1586.

26 Menon B, Dogra V, Nima G, et al. Evaluation of thoracic HRCT findings in sarcoidosis. Eur Respir J 2014; 44: Suppl. 58, 664 .

27 Papiris S, Georgakopoulos A, Pianou N, et al. ${ }^{18} \mathrm{~F}-\mathrm{FDG}-\mathrm{PET} / \mathrm{CT}$ for the evaluation of sarcoidosis patients. Eur Respir J 2014; 44: Suppl. 58, 657.

28 Stanton K, Ganigara M, Corte T, et al. The utility of cardiac magnetic resonance imaging in the diagnosis of cardiac sarcoidosis. Eur Respir J 2014; 44: Suppl. 58, 1728.

29 Porakhonko N, Lapteva I, Kharevich O, et al. Factors of cardiac dysfunction in advanced COPD. Eur Respir J 2014; 44: Suppl. 58, 537.

30 Galban C, Boes J, Hoff B, et al. Evaluation of parametric response mapping for monitoring COPD subtypes. Eur Respir J 2014; 44: Suppl. 58, 642.

31 Jobst B, Triphan S, Sedlaczek O, et al. Functional lung MRI in COPD: comparative assessment of T1 mapping, oxygen-enhanced T1 mapping and perfusion MRI at 1.5 Tesla. Eur Respir J 2014; 44; Suppl. $58,1723$.

32 Parra-Robles J, Vasilescu D, Hogg JC, et al. Modelling of ${ }^{3} \mathrm{He}$ gas diffusion in realistic $3 \mathrm{D}$ models of human acinar airways obtained from micro-CT images. Eur Respir J 2014; 44; Suppl. 58, 4972.

33 Hart K, Marshall H, Stewart N, et al. Quantitative lung T1 mapping and oxygen enhanced MRI in IPF. Eur Respir J 2014; 44: Suppl. 58, 1727.

34 Stewart N, Leung G, Norquay G, et al. Age-dependent changes in whole-lung septal thickness measured by ${ }^{129}$ Xe MRI: a comparison of healthy volunteers and subjects with IPF. Eur Respir J 2014; 44: Suppl. 58, 550.

35 De Backer J, Vos W, Smaldone G, et al. Disease progression in IPF assessed using pulmonary function tests and functional respiratory imaging (FRI) - a pilot study. Eur Respir J 2014; 44: Suppl. 58, 654.

36 Slebos DJ, Klooster K, Koegelenberg C, et al. Efficacy of targeted lung denervation in patients with moderate to severe COPD. Eur Respir J 2014; 44: Suppl. 58, 1774s.

37 Slebos DJ, Klooster K, Koegelenberg C, et al. Safety and feasibility of targeted lung denervation in patients with moderate to severe COPD. Eur Respir J 2014; 44: Suppl. 58, 3720s.

38 Valipour A, Pison C, Kessler R, et al. Bilateral targeted lung denervation in patients with COPD in a single procedure. Eur Respir J 2014; 44: Suppl. 58, 1775s.

39 Zoumot Z, Lo Maoro A, Aliverti A, et al. Successful lung volume reduction improves inspiratory paradoxical movement of the lower rib cage at rest as measured by optoelectronic plethysmography. Eur Respir J 2014; 44: Suppl. 58, 3718s.

40 Lador F, Hachulla AL, Espinosa V, et al. Pulmonary perfusion after endoscopic reduction of emphysema. Eur Respir J 2014; 44: Suppl. 58, 3708s.

41 Hartman JE, Klooster K, ten Hacken NHT, et al. Long-term follow up after bronchoscopic lung volume reduction coil treatment. Eur Respir J 2014; 44: Suppl. 58, 1777s.

42 Davey C, Zoumot Z, McNulty W, et al. Bronchoscopic lung volume reduction with endobronchial valves for patients with heterogeneous emphysema and intact interlobar fissures (BeLieVeR-HIFi). Eur Respir J 2014; 44: Suppl. 58, 1773s.

43 Eberhardt R, Gerovasili V, Kontogianni K, et al. Effect of endoscopic lung volume reduction on established pulmonary hypertension in severe emphysema patients. Eur Respir J 2014; 44: Suppl. 58, 3711s.

44 Lepper PM, Fähndrich S, Trudzinski F, et al. Endoscopic lung volume reduction in patients with very low FEV1. Eur Respir J 2014; 44: Suppl. 58, 3712s.

45 Herzog D, Hippenstiel S, Schürmann D, et al. Strict bed rest after EBV implant reduces pneumothorax rate. Eur Respir J 2014; 44: Suppl. 58, 3737s.

46 Bakeer M, Abd El-Gawad T, El-Metwaly Ali R, et al. Low cost biological lung volume reduction therapy for advanced emphysema. Eur Respir J 2014; 44: Suppl. 58, 3705s.

47 Marchetti N, Krahnke J, Kaufman T, et al. Treatment of advanced homogenous emphysema with endobronchial coils compared to NETT subjects who received LVRS or medical therapy. Eur Respir J 2014; 44: Suppl. $58,4638$.

48 Gupta D, Dadhwal DS, Agarwal R, et al. Endobronchial ultrasound-guided transbronchial needle aspiration vs conventional transbronchial needle aspiration in the diagnosis of sarcoidosis. Chest 2014; 146: 547-556.

49 Goyal A, Gupta D, Agarwal R, et al. Value of different bronchoscopic sampling techniques in diagnosis of sarcoidosis: a prospective study of 151 patients. J Bronchology Interv Pulmonol 2014; 21: 220-226.

50 Plit ML, Havryk AP, Hodgson A, et al. Rapid cytological analysis of endobronchial ultrasound-guided aspirates in sarcoidosis. Eur Respir J 2013; 42: 1302-1308.

51 Rozman A, Marc Malovrh M. Elastography strain ratio in the staging of mediastinal lymph nodes. Eur Respir J 2014; 44: Suppl. 58, 3562.

52 Herth FJF, Gompelmann D, Hoffmann H, et al. Bronchoscopic trans-parenchymal nodule access (BTPNA) - a new real-time image-guided approach to accessing solitary pulmonary nodules (SPNs) - first in human data. Eur Respir J 2014; 44: Suppl. 58, 692.

53 Walsh SL, Wells AU, Sverzellati N, et al. An integrated clinicoradiological staging system for pulmonary sarcoidosis: a case-cohort study. Lancet Respir Med 2014; 2: 123-130. 
54 Wijnen PA, Cremers JP, Nelemans PJ, et al. Association of the TNF- $\alpha$ G-308A polymorphism with TNF-inhibitor response in sarcoidosis. Eur Respir J 2014; 43: 1730-1739.

55 Sweiss NJ, Noth I, Mirsaeidi M, et al. Efficacy results of a 52-week trial of adalimumab in the treatment of refractory sarcoidosis. Sarcoidosis Vasc Diffuse Lung Dis 2014; 31: 46-54.

56 Judson MA, Baughman RP, Costabel U, et al. Safety and efficacy of ustekinumab or golimumab in patients with chronic sarcoidosis. Eur Respir J 2014; 44: 1296-1307.

57 Ryerson CJ, Corte TJ, Collard HR, Richeldi L, et al. A global registry for idiopathic pulmonary fibrosis: the time is now. Eur Respir J 2014; 44: 273-276.

58 King TE Jr, Bradford WZ, Castro-Bernardini S, et al. A phase 3 trial of pirfenidone in patients with idiopathic pulmonary fibrosis. N Engl J Med 2014; 370: 2083-2092.

59 Costabel U, Albera C, Bradford WZ, et al. Analysis of lung function and survival in RECAP: An open-label extension study of pirfenidone in patients with idiopathic pulmonary fibrosis. Sarcoidosis Vasc Diffuse Lung Dis 2014; 31: 198-205.

60 Richeldi L, du Bois RM, Raghu G, et al. Efficacy and safety of nintedanib in idiopathic pulmonary fibrosis. $N$ Engl J Med 2014; 370: 2071-2082.

61 Corte TJ, Keir GJ, Dimopoulos K, et al. BPHIT Study Group. Bosentan in pulmonary hypertension associated with fibrotic idiopathic interstitial pneumonia. Am J Respir Crit Care Med 2014; 190: 208-217.

62 Saketkoo LA, Mittoo S, Huscher D, et al. Connective tissue disease related interstitial lung diseases and idiopathic pulmonary fibrosis: provisional core sets of domains and instruments for use in clinical trials. Thorax 2014; 69: 428-436.

63 Migita K, Arai T, Jiuchi Y, et al. Predictors of mortality in patients with interstitial lung disease treated with corticosteroids: results from a cohort study. Medicine (Baltimore) 2014; 93: e175.

64 Kocks J, Weatherall M, Metting E, et al. Phenotyping airways disease by cluster analysis in primary care: 6 distinct clusters identified. Eur Respir J 2014; 44: Suppl. 58, 3837.

65 Van Gemert F, Kirenga B, Chavannes N, et al. Prevalence of COPD in a sub-Saharan country: Fresh Air Uganda survey. Eur Respir J 2014; 44: Suppl. 58, 2207.

66 Jones R, Price D, Lee, et al. The GOLD COPD categories are inaccurate in predicting future risk in a UK primary care dataset and the 2014 changes to cut-off values have not helped. Eur Respir J 2014; 44: Suppl. 58, 3642.

67 Price D, Rigazio A, Postma D, et al. Blood eosinophilia and the number of exacerbations in COPD patients. Eur Respir J 2014; 44: Suppl. 58, 2165.

68 Sundh J, Ställberg B Lisspers K, et al. What influences change in DOSE index in a seven year follow-up? Eur Respir J 2014; 44: Suppl. 58, 703.

69 Jones RC, Donaldson GC, Chavannes NH, et al. Derivation and validation of a composite index of severity in chronic obstructive pulmonary disease: the DOSE Index. Am J Respir Crit Care Med 2009; 180: 1189-1195.

70 Kruis A, Boland M, Assendelft W, et al. Is integrated disease management of COPD effective? Results of the RECODE cluster-randomized controlled trial in real world patients. Eur Respir J 2014; 44: Suppl. 58, 2581.

71 Francesqui J, Sellarés J, Soler N, et al. Integrated care model between pneumology and primary care: Impact in respiratory diseases control. Eur Respir J 2014; 44: Suppl. 58, 1979. 